\title{
Data-Driven Affine Deformation Estimation and Correction in Cone Beam Computed Tomography
}

\author{
Vincent Van Nieuwenhove, Jan De Beenhouwer, Thomas De Schryver, Luc Van Hoorebeke, and Jan Sijbers
}

\begin{abstract}
In computed tomography (CT), motion and deformation during the acquisition lead to streak artefacts and blurring in the reconstructed images. To remedy these artefacts, we introduce an efficient algorithm to estimate and correct for global affine deformations directly on the cone beam projections. The proposed technique is data driven and thus removes the need for markers and/or a tracking system. A relationship between affine transformations and the cone beam transform is proved and used to correct the projections. The deformation parameters that describe deformation perpendicular to the projection direction are estimated for each projection by minimizing a plane-based inconsistency criterion. The criterion compares each projection of the main scan with all projections of a fast reference scan, which is acquired prior or posterior to the main scan. Experiments with simulated and experimental data show that the proposed affine deformation estimation method is able to substantially reduce motion artefacts in cone beam CT images.
\end{abstract}

Index Terms-Computed tomography, CBCT, image reconstruction, motion correction.

\section{INTRODUCTION}

$\mathbf{I}$ N COMPUTED tomography (CT), conventional reconstruction algorithms assume that the scanned object does not move or deform during the acquisition. However, there is often voluntary or involuntary motion. Voluntary motion is caused by a deliberately applied process that changes the size of the object while scanning, such as compression/expansion of foam or heating of bakery products (e.g., rising of bread during the baking process). Involuntary motion is any motion that occurs unintentionally, such as shrinkage during drying of the object, sudden movement, or natural motion such as cardiac or respiratory motion. Tomographic reconstruction algorithms that do not account for these types of motion suffer from motion artefacts in the reconstructed images such as blurring or streaking.

An effective way to reduce motion artefacts is to simply reduce the scan time to limit (the risk of) sample deformation

Manuscript received April 8, 2016; revised October 10, 2016; accepted January 4, 2017. Date of publication January 15, 2017; date of current version February 3, 2017. This work was supported in part by the Fund for Scientific Research-Flanders (FWO) under Grant S004217N and in part by the Institute for the Promotion of Innovation through Science and Technology (IWT-Vlaanderen) under Grant SBO12033. The associate editor coordinating the review of this manuscript and approving it for publication was Dr. Abd-Krim Karim Seghouane.

V. Van Nieuwenhove, J. De Beenhouwer, and J. Sijbers are with the Imec-Vision Lab, Department of Physics, University of Antwerp, B-2610 Antwerp, Belgium (e-mail: Vincent.VanNieuwenhove@uantwerpen.be Jan.DeBeenhouwer@uantwerpen.be; Jan.Sijbers@uantwerpen.be).

T. De Schryver and L. Van Hoorebeke are with Department of Physics and Astronomy, Ghent University, B-9000 Ghent, Belgium (e-mail: Thomas.Deschryver@ugent.be; Luc.Van.Hoorebeke@ugent.be).

Color versions of one or more of the figures in this paper are available online at http://ieeexplore.ieee.org.

Digital Object Identifier 10.1109/TIP.2017.2651370 during the acquisition [1], [2]. Faster scanning, however, inevitably leads to reduced signal-to-noise ratio (SNR) of the reconstructed images. Especially in micro-CT imaging, where the X-ray flux is much smaller compared to clinical CT, scan times of the order of hours are common to obtain sufficient SNR and spatial resolution. Hence, many micro-CT experiments suffer from a high risk of sample motion/deformation during the acquisition, which significantly reduces the spatial resolution of the reconstructed images. Hence, there is a clear need for reconstruction methods that can generate high quality images from motion corrupted scans.

Several image reconstruction methods have been developed to reduce motion artefacts. A first class of methods rearranges projection images of multiple gantry rotations in different subsets, after which each subset is independently reconstructed. Gated CT algorithms, for example, are mostly used with periodic deformation. These algorithms sort projections into subsets, based on, for example, the phase in the cardiac or respiratory cycle [3], [4]. If the deformation is slow and nonperiodic, subsequent scans can be acquired where each of the scans can be regarded as a motionless subset.

As a result, the reconstructions of these subsets will be almost motion artefact free. The time resolution of these techniques can be improved by shortening the acquisition time of a single scan by decreasing the exposure time and/or the number of projections [2]. However, a reduction of the exposure time inevitably leads to a decrease in SNR. Moreover, a reduction of the number of projections per scan results in undersampling artefacts.

A second class of motion artefact reduction methods directly models known motion in the reconstruction algorithm. Analytic algorithms such as Filtered Back Projection (FBP) or Feldkamp (FDK), and algebraic reconstruction algorithms, such as the Algebraic Reconstruction Technique (ART), have been proposed to include an a priori known deformation model [5], [6]. Roux et al., for example, introduced a 2D exact reconstruction method for objects deforming in time by a known affine transformation [7], this method was generalised to a broader range of deformations by Desbat et al. [8].

While several approaches are able to correct for deformation with a known deformation model and parameters, the true deformation parameters are still unknown. Markers and tracking systems can greatly facilitate motion estimation [9], [10] but suffer from inherent disadvantages. Firstly, marker placement is time consuming, since they have to be placed very carefully to avoid damaging the sample. Moreover, markers may shift during the acquisition. Secondly, a specialized and often costly tracking system is needed. 
On the other hand, data driven estimation of the motion model parameters is still a difficult and time consuming procedure. For this purpose, some multiple gantry rotation approaches combine motion model based reconstruction with gated CT. Thereby, a deformation model is estimated by means of volume registration of neighboring and motion artefact free cardiac/respiratory phases. The estimated deformation model then allows exploiting projections of other time points in the reconstruction of a specific time point without introducing motion artefacts [11]-[13]. These techniques, however, require the quality of the images at the individual time frames to be high (i.e., free from motion artefacts and sufficiently high SNR), which limits their applicability.

Data driven motion/deformation estimation methods that only rely on data acquired during a single gantry rotation have also been proposed. Most of these techniques, however, assume 2D parallel or fan beam projections [14], [15]. For example, the effect of affine transformations on the 2D radon transform was studied in [7] and [16]-[18]. Frysch et al. estimated rigid motion directly on cone beam projection images [19], [20]. A fan beam and cone beam motion correction without deformation model was proposed by Leng et al. [21]. It estimates a motion corrected version of the motion contaminated projections based on the rest of the projections. However, this method assumes that a large portion of the projections are not corrupted by motion.

In this work, we introduce a 3D estimation and correction algorithm for global affine deformation, which works directly on the cone beam projections. The correction of the affine deformation is achieved by exploiting the relationship between cone beam projections and affine transformations. To estimate the affine deformation parameters, a data driven approach is proposed that estimates the deformation parameters of each individual cone beam projection with respect to a fast reference scan. The estimation of the deformation parameters is performed directly in the projection domain, avoiding time consuming reconstructions. To achieve this, an inconsistency criterion, based on the exact reconstruction algorithm of Grangeat [22] and Defrise and Clack [23], is minimized by a non-convex optimization procedure. A similar inconsistency criterion was recently introduced to compensate for rigid motion [19], [24]-[27].

The paper is organized as follows. Some definitions are introduced in Section II-A. Section II-B describes the relation between the cone beam projections and affine transformations which allows to correct for affine deformations directly in the projection domain. Next, a method to estimate affine deformation parameters directly in the projection domain is proposed. Section III describes phantom and real data experiments, the results of which are presented in Section IV. Section V concludes the paper.

\section{METHOD}

\section{A. Definitions}

1) Cone Beam Geometry: In X-ray $\mathrm{CT}$, each acquired projection is associated with a projection geometry: the source position $\mathbf{s}$ and the position of the individual detector elements. The detector is assumed to be rectangular and flat with the

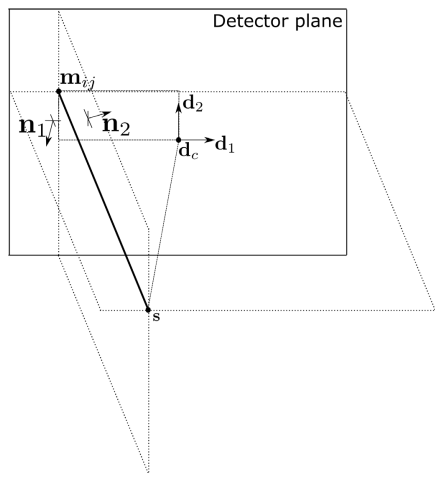

Fig. 1. Schematic overview of the geometry of a cone beam projection.

detector center at position $\mathbf{d}_{c}$. The position $\mathbf{m}_{i j}$ of the detector element at detector coordinates $(i, j)$ can then be described as: $\mathbf{m}_{i j}=\mathbf{d}_{c}+i \cdot \mathbf{d}_{1}+j \cdot \mathbf{d}_{2}$, with $\mathbf{d}_{1}$ being the $3 \mathrm{D}$ vector from detector element $(0,0)$ to $(1,0), \mathbf{d}_{2}$ the $3 \mathrm{D}$ vector from detector element $(0,0)$ to $(0,1), i \in[-I / 2, I / 2]$ and $j \in[-J / 2, J / 2]$, where $I$ and $J$ are the number of detector pixels horizontally and vertically, respectively. The projection geometry of a single projection can thus be described with the following set of vectors $G=\left\{\mathbf{s}, \mathbf{d}_{c}, \mathbf{d}_{1}, \mathbf{d}_{2}\right\}$.

For each ray, two planes can be defined that both contain the vector $\mathbf{m}_{i j}-\mathbf{s}$ and are parallel to $\mathbf{d}_{1}$ or $\mathbf{d}_{2}$.

The normals to these planes, $\mathbf{n}_{1}(j)$ and $\mathbf{n}_{2}(i)$, are given by:

$$
\begin{aligned}
& \mathbf{n}_{1}(j)=\frac{\left(\mathbf{m}_{i j}-\mathbf{s}\right) \times \mathbf{d}_{1}}{\left\|\left(\mathbf{m}_{i j}-\mathbf{s}\right) \times \mathbf{d}_{1}\right\|} \\
& \mathbf{n}_{2}(i)=\frac{\left(\mathbf{m}_{i j}-\mathbf{s}\right) \times \mathbf{d}_{2}}{\left\|\left(\mathbf{m}_{i j}-\mathbf{s}\right) \times \mathbf{d}_{2}\right\|},
\end{aligned}
$$

with $\|$.$\| denoting the Euclidean norm (2-norm). A cone beam$ projection of a function $f(\mathbf{x})\left(\mathbf{x} \in \mathbb{R}^{3}\right)$ can then be described as:

$$
\begin{aligned}
C_{G}(f(\mathbf{x}), i, j)= & \int f(\mathbf{x}) \delta\left(\mathbf{n}_{1}(j) \cdot(\mathbf{x}-\mathbf{s})\right) \\
& \times \delta\left(\mathbf{n}_{2}(i) \cdot(\mathbf{x}-\mathbf{s})\right) d \mathbf{x} .
\end{aligned}
$$

The overall geometry is visualized in Fig. 1. During a CT acquisition, multiple projections are acquired, each corresponding to a specific source and detector position.

2) Affine Transformation: An affine transformation is a combination of translations, rotations, differential scalings and shearings. An affine transformed volume $f_{T}(\mathbf{x})$ of $f(\mathbf{x})$ can be calculated as follows:

$$
f_{T}(\mathbf{x})=f(\mathbf{A x}+\mathbf{u}),
$$

where $\mathbf{A}$ is a $3 \times 3$ linear map and $\mathbf{u}$ a $3 \times 1$ vector describing translation.

\section{B. Affine Transformation and Cone Beam Projections}

The effect of an affine transformation on the 2D Radon transform has been well studied [7], [16], [17]. In this section, we will elaborate on the extension to cone beam projections.

Let $C_{G}\left(f_{T}(\mathbf{x}), i, j\right)$ be a cone beam projection of an affine transformed object $f_{T}(\mathbf{x})$ associated with the projection geometry $G$. Then, $C_{G}\left(f_{T}(\mathbf{x}), i, j\right)$ can be transformed to a cone 
beam projection of the non-deformed object $f(\mathbf{x})$, as follows (proof in Appendix):

$$
\begin{aligned}
& C_{G^{\prime}}(f(\mathbf{x}), i, j) \\
& \quad=\frac{\left\|\left(\mathbf{A}^{-1}\right)^{T} \mathbf{n}_{1}(j)\right\|\left\|\left(\mathbf{A}^{-1}\right)^{T} \mathbf{n}_{2}(i)\right\|}{\operatorname{det}\left(\mathbf{A}^{-1}\right)} C_{G}\left(f_{T}(\mathbf{x}), i, j\right),
\end{aligned}
$$

associated with virtual projection geometry $G^{\prime}=$ $\left\{\mathbf{s}^{\prime}, \mathbf{d}_{c}^{\prime}, \mathbf{d}_{1}^{\prime}, \mathbf{d}_{2}^{\prime}\right\}$, where:

$$
\left\{\begin{array}{l}
\mathbf{s}^{\prime}=\mathbf{A s}+\mathbf{u} \\
\mathbf{d}_{c}^{\prime}=\mathbf{A} \mathbf{d}_{c}+\mathbf{u} \\
\mathbf{d}_{1}^{\prime}=\mathbf{A} \mathbf{d}_{1} \\
\mathbf{d}_{2}^{\prime}=\mathbf{A} \mathbf{d}_{2}
\end{array}\right.
$$

If all projections of a CT dataset are transformed to be consistent with projections of the non-deformed object, then a $3 \mathrm{D}$ image that is free of motion artefacts can be reconstructed.

\section{Estimation of Motion/Deformation Parameters}

In Section II-B, a procedure was described to correct a cone beam projection of an affine transformed object, which requires knowledge of that affine transformation. In a real experiment, the deformation parameters are unknown and need to be estimated from the measured projection data. In this section, an approach to automatically estimate the affine deformation parameters is introduced.

The section starts with the general data acquisition and estimation strategy (section II-C.1) followed by the concept of redundantly measured planes (section II-C.2). Based on this concept, an objective function is derived in section II-C.3. Finally, a strategy to optimize this objective function is introduced.

1) Estimation Strategy: To ensure accurate deformation estimation, we propose the following acquisition protocol. The complete acquisition consists of two parts:

Main scan: Conventional scan with $N$ projections.

Reference scan: A short $360^{\circ}$ equiangular scan with $N_{\text {ref }}$ projections $\left(N_{\text {ref }} \ll N\right)$ that is acquired immediately before or after the main scan (see Fig. 2a). Reference projections in multiple directions are required in order to estimate the deformation parameters of projections, of the main scan, in different directions. During the reference scan, the object is assumed to be motionless, which is a reasonable assumption since this scan is acquired in a very short time span. Reference scans have a limited extra cost and are often already implemented in commercial high resolution micro-CT scanners [28]. In practice, an angular step of $45^{\circ}$ was observed to be sufficient.

For each projection of the main scan, an affine deformation has to be estimated with respect to the reference scan. Affine deformation parameters corresponding to a certain projection of the main scan can be estimated by minimizing a criterion that quantifies the inconsistency between that projection and all projections of the reference scan.

Unfortunately, not all affine parameters corresponding to a single projection can be accurately estimated. For example, a

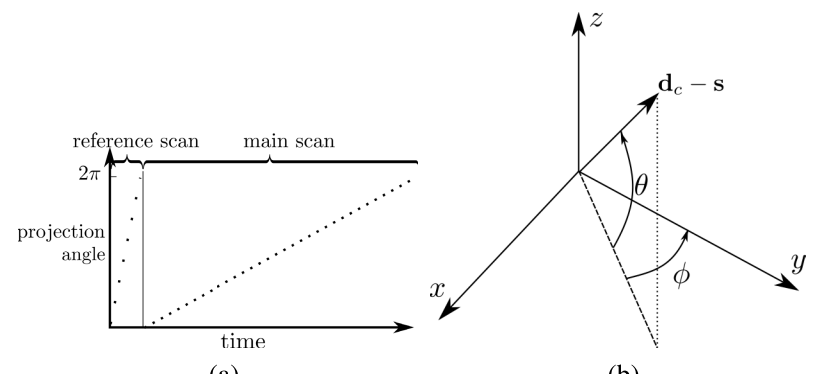

(a)

(b)

Fig. 2. (a) Acquisition protocol: A short reference scan followed by the main scan. The order of both scans can be changed. (b) The angles $\theta$ and $\phi$ that define the rotation of the coordinate system.

translation in the projection direction (i.e., parallel to $\mathbf{d}_{c}-\mathbf{s}$ ) will be almost indistinguishable from a scaling of the object. To overcome this problem, only parameters that describe deformations perpendicular to the projection direction will be estimated. To that end, we introduce a change of the coordinate system for the current projection. The coordinate system is rotated to align the projection direction $\mathbf{d}_{c}-\mathbf{s}$ with one of the coordinate axes. This is achieved by multiplying the vectors associated with projection geometry $G$ with a rotation matrix $\mathbf{R}$. In our work, without loss of generality, the projection direction is rotated such that it becomes parallel to the $\mathbf{y}$-axis. Hence, $\mathbf{R}$ is written as:

$$
\mathbf{R}=\mathbf{R}_{x} \mathbf{R}_{z}
$$

with $\mathbf{R}_{x}$ and $\mathbf{R}_{z}$ a rotation around the $\mathrm{x}$-axis and $\mathrm{z}$-axis, respectively:

$$
\begin{aligned}
\mathbf{R}_{x}= & {\left[\begin{array}{ccc}
1 & 0 & 0 \\
0 & \cos \theta & \sin \theta \\
0 & -\sin \theta & \cos \theta
\end{array}\right] } \\
\mathbf{R}_{z}= & {\left[\begin{array}{ccc}
\cos \phi & \sin \phi & 0 \\
-\sin \phi & \cos \phi & 0 \\
0 & 0 & 1
\end{array}\right], }
\end{aligned}
$$

where the angles $\theta$ and $\phi$ are given by (see Fig. 2):

$$
\begin{aligned}
& \theta=\operatorname{sgn}\left(d_{c, z}-s_{z}\right) \arccos \left(\frac{\sqrt{\left(d_{c, x}-s_{x}\right)^{2}+\left(d_{c, y}-s_{y}\right)^{2}}}{\left\|\mathbf{d}_{c}-\mathbf{s}\right\|}\right) \\
& \phi=\arctan \left(\frac{d_{c, x}-s_{x}}{d_{c, y}-s_{y}}\right) .
\end{aligned}
$$

with sgn the sign function, arctan the four-quadrant inverse tangent and $d_{c}$, and $s$. the components of the vector $\mathbf{d}_{c}$ and $\mathbf{s}$, respectively.

Hence, the projection geometry in the rotated coordinate system is given by:

$$
G_{\mathbf{R}}=\left\{\mathbf{s}_{\mathbf{R}}, \mathbf{d}_{c, \mathbf{R}}, \mathbf{d}_{1, \mathbf{R}}, \mathbf{d}_{2, \mathbf{R}}\right\}=\left\{\mathbf{R s}, \mathbf{R d}_{c}, \mathbf{R d}_{1}, \mathbf{R d}_{2}\right\} .
$$

The subscript $\cdot \mathbf{R}$ denotes that the variable is expressed in the rotated coordinate system. The affine deformation in the rotated coordinate system is given by:

$$
\left\{\mathbf{A}_{\mathbf{R}}, \mathbf{u}_{\mathbf{R}}\right\}=\left\{\mathbf{R A R}^{-1}, \mathbf{R u}\right\}
$$


The rotation of the coordinate system allows extracting parameters that describe deformations perpendicular to the projection axis (i.e., the y-axis). Let $\mathbf{q}_{k}$ denote the set of parameters in the rotated coordinate system characterizing the deformation of projection $k$ perpendicular to the $\mathrm{y}$-axis:

$$
\mathbf{q}_{k}=\left[\begin{array}{llllll}
a_{\mathbf{R}, x, x} & a_{\mathbf{R}, x, z} & a_{\mathbf{R}, z, x} & a_{\mathbf{R}, z, z} & u_{\mathbf{R}, x} & u_{\mathbf{R}, z}
\end{array}\right]^{T},
$$

with $a_{\mathbf{R}, .,}$ an element of the matrix $\mathbf{A}_{\mathbf{R}}$ and $u_{\mathbf{R}, \text {, an element }}$ of the vector $\mathbf{u}_{\mathbf{R}}$. For each projection $k$, the parameters of the vector $\mathbf{q}_{k}$ are estimated by minimizing an objective function $F_{k}$ that quantifies the inconsistency of the $k^{\text {th }}$ projection with respect to the reference scan:

$$
\mathbf{q}_{k}^{*}=\arg \min _{\mathbf{q}_{k}} F_{k}\left(\mathbf{q}_{k}\right) .
$$

The proposed objective function will be derived in section II-C.3.

2) Redundantly Measured Planes: During the reference and the main scan, many planes are scanned twice. All planes that are sampled by the $k^{\text {th }}(k=1, \ldots, N)$ projection of the main scan and the $l^{\text {th }}\left(l=1, \ldots, N_{r e f}\right)$ projection of the reference scan contain the source positions $\mathbf{s}_{k}$ and $\mathbf{s}_{l}$. These two points define a line $\mathbf{s}_{l}+\alpha\left(\mathbf{s}_{k}-\mathbf{s}_{l}\right)$ with $\alpha \in \mathbb{R}$ that defines the sheaf of all planes that are sampled by both projections.

The point $\mathbf{c}_{k, l}=\mathbf{d}_{c, k}+i_{k, l} \mathbf{d}_{1, k}+j_{k, l} \mathbf{d}_{2, k}$, where the line $\mathbf{s}_{l}+\alpha\left(\mathbf{s}_{k}-\mathbf{s}_{l}\right)$ intersects with the detector plane $k$, can be calculated by solving the following system of equations for $i_{k, l}, j_{k, l}$ and $\alpha_{k}$ :

$$
\left[\begin{array}{lll}
\mathbf{d}_{1, k} & \mathbf{d}_{2, k} & \left(\mathbf{s}_{k}-\mathbf{s}_{l}\right)
\end{array}\right]\left[\begin{array}{c}
i_{k, l} \\
j_{k, l} \\
-\alpha_{k}
\end{array}\right]=\mathbf{s}_{l}-\mathbf{d}_{c, k},
$$

with $\alpha_{k}$ the value of the parameter $\alpha$ where the line intersects with the detector plane. The same procedure can be repeated to calculate $\mathbf{c}_{l, k}$, the point where the line $\mathbf{s}_{l}+\alpha\left(\mathbf{s}_{k}-\mathbf{s}_{l}\right)$ intersects with the detector plane $l$.

Let $\lambda$ be a plane sampled by both the $k^{\text {th }}$ main projection and a reference projection. The projection of $\lambda$ on the main projection then corresponds to a line defined by $\mathbf{c}_{k, l}$ and a unit vector $\mathbf{l}_{k}(\zeta)=\cos (\zeta) \frac{\mathbf{d}_{1, k}}{\left\|\mathbf{d}_{1, k}\right\|}+\sin (\zeta) \frac{\mathbf{d}_{2, k}}{\left\|\mathbf{d}_{2, k}\right\|}$ in the detector plane (with $\zeta$ the angle between $\mathbf{l}_{k}$ and $\mathbf{d}_{1, k}$ ).

As a result, any plane, sampled by both the main and the reference projection, is parametrized by the angle $\zeta$.

The vector $\mathbf{l}_{l}$, describing the projection of the plane on the $l^{\text {th }}$ reference detector, should lie in the same plane as $\mathbf{l}_{k}$. Hence, $\mathbf{l}_{l}$ can be determined as follows:

$$
\mathbf{l}_{l}(\zeta)=\frac{1}{Z}\left(\left(\mathbf{d}_{1, l} \times \mathbf{d}_{2, l}\right) \times \mathbf{n}_{\lambda}\right)
$$

with $\mathbf{n}_{\lambda}(\zeta)=\frac{1}{Z^{\prime}} \mathbf{l}_{k}(\zeta) \times\left(\mathbf{s}_{l}-\mathbf{s}_{k}\right)$, the normal to the plane and $Z$ and $Z^{\prime}$ normalization constants. A graphical overview of the geometry of a plane sampled by the $k^{\text {th }}$ projection of the main scan and the $l^{\text {th }}$ projection of the reference scan is shown in Fig. 3.

The angular range of $\zeta$ for which the corresponding planes have an actual intersection with the detector support of two projections is highly dependent on the direction of the projections. If two projections lie approximately opposite to each

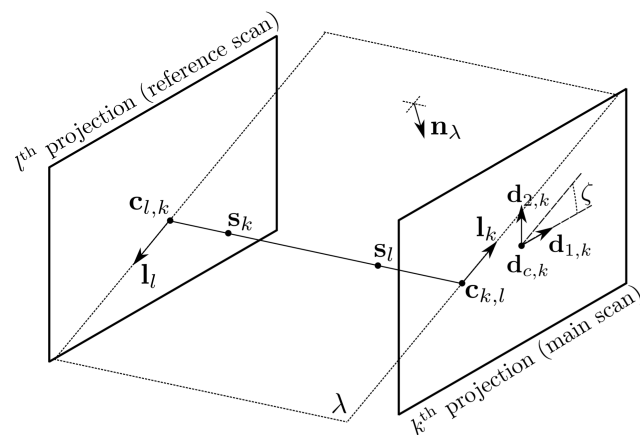

Fig. 3. The parameters of a plane sampled by both the $k^{\text {th }}$ main and the $l^{\text {th }}$ reference projection.

other, the point $\mathbf{c}_{k, l}$ lies on the detector. As a result, all planes, sampled by both the main and the reference projection, have an actual intersection with the detector support. On the other hand, if the directions of the two projections are approximately the same, only a small angular range of $\zeta$ will correspond to planes intersecting with the actual detector support. A more in depth discussion can be found in [27].

3) Objective Function: To assess the consistency of a projection with the reference scan, a cone beam inconsistency criterion is defined that is based on redundantly measured planes (see section II-C.2). The inconsistency criterion is based on the exact cone beam reconstruction method of Grangeat and compares the derivative of the 3D Radon transform of redundantly measured planes in the reconstruction domain directly on the projections [22], [23]. As such, it avoids computationally intensive reconstructions to evaluate the estimated deformation parameters. The optimization of this criterion was proposed in several recent techniques to estimate rigid motion and geometric system parameters [19], [24], [25], [27], [29]. Although the criterion is theoretically restricted to nontruncated data, it has been successfully applied to truncated data as well [25], [29]. The connection with the epipolar geometry was established in [27] and [26].

A plane $\lambda$, sampled by a projection associated with projection geometry $G$, containing the source position $\mathbf{s}$ and a point on the detector $\mathbf{m}_{i j}$ is projected as a line on the detector: $\mathbf{y}(v)=v \mathbf{l}(\zeta)+\mathbf{m}_{i j}$. From the data on this line, the value of the radial derivative of the 3D Radon transform corresponding to the plane $\lambda$ can be calculated as follows:

1) In the first step, the projection undergoes an inverse cosine weighting, similar to the well-known Feldkamp, David and Kress (FDK) algorithm [23], [30]:

$$
E\left(C_{G}, i, j\right)=\frac{C_{G}(f(\mathbf{x}), i, j)}{|\mathbf{w} \cdot \mathbf{t}(i, j)|}
$$

with $\mathbf{t}(i, j)=\frac{1}{Z}\left(\mathbf{m}_{i j}-\mathbf{s}\right)$ the unit vector in the direction of the ray intersecting a detector element at position $(i, j)$ on the projection with projection geometry $G$ and $\mathbf{w}=\frac{1}{Z^{\prime}} \mathbf{d}_{1} \times \mathbf{d}_{2}$ the normal to the detector. $Z$ and $Z^{\prime}$ are normalization constants.

2) In the second step, the cone beam projections are integrated along the projection of the plane 


$$
\begin{aligned}
& \left(\mathbf{y}(v)=v \mathbf{l}(\zeta)+\mathbf{m}_{i j}\right): \\
& \quad \begin{aligned}
L\left(C_{G}, i, j, \mathbf{l}\right) \\
\quad=\int_{-\infty}^{+\infty} E\left(C_{G}, i+v \mathbf{l} \cdot \mathbf{d}_{1}, j+v \mathbf{l} \cdot \mathbf{d}_{2}\right) d v .
\end{aligned}
\end{aligned}
$$

3) Finally, a differentiation in the direction perpendicular to $\mathbf{I}$ is performed, resulting in the radial derivative of the 3D radon transform of the scanned object:

$$
H\left(C_{G}, i, j, \mathbf{l}\right)=\nabla_{\mathbf{r}} L\left(C_{G}, i, j, \mathbf{l}\right),
$$

with $\mathbf{r}=(\mathbf{w} \times \mathbf{l}) /\|\mathbf{w} \times \mathbf{l}\|$. The radial derivative is approximated with the central difference method.

The domain of the radial derivative of the 3D radon transform can only partially be calculated since only a limited set of planes in the reconstruction domain is sampled; that is, only planes that are sampled by the cone beam, defined by projection geometry $G$, are sampled. Since the goal of the objective function is to compare planes that are sampled by the $k^{\text {th }}$ projection of the main scan and $l^{\text {th }}$ projection of the reference scan, only the planes containing the point $\mathbf{c}_{k, l}$ will be considered: $H\left(C_{G_{k}}, i_{k, l}, j_{k, l}, \mathbf{l}\right)=H\left(C_{G_{k}}, \mathbf{c}_{k, l}, \mathbf{l}\right)$. The value of the derivative of the $3 \mathrm{D}$ radon transform corresponding to a plane calculated on two different projections should be the same. As a result, the difference between these derivatives should, theoretically, be zero. An intermediate inconsistency function $T_{k, l}$ is defined by repeating this procedure for different planes, which are all elements of the sheaf of planes defined by the line $\mathbf{s}_{l}+\alpha\left(\mathbf{s}_{k}-\mathbf{s}_{l}\right)$ :

$$
\begin{aligned}
T_{k, l}\left(\mathbf{q}_{k}\right)= & \sum_{\left\{\mathbf{l}_{k}(\zeta): \zeta \in[0, \pi)\right\}}\left[H\left(C_{G_{\mathbf{R}, k}}^{\prime}\left(\mathbf{q}_{k}\right), \mathbf{c}_{k, l}\left(\mathbf{q}_{k}\right), \mathbf{l}_{k}\right)\right. \\
& \left.-H\left(C_{G_{\mathbf{R}, l}}, \mathbf{c}_{l, k}\left(\mathbf{q}_{k}\right), \mathbf{l}_{l}\left(\mathbf{q}_{k}\right)\right)\right]^{2},
\end{aligned}
$$

with:

$$
\begin{aligned}
& C_{G_{\mathbf{R}, k}}^{\prime}\left(\mathbf{q}_{k}, i, j\right) \\
& =\frac{\left\|\left(\mathbf{A}\left(\mathbf{q}_{\mathbf{k}}\right)^{-1}\right)^{T} \mathbf{n}_{1}(j)\right\|\left\|\left(\mathbf{A}\left(\mathbf{q}_{\mathbf{k}}\right)^{-1}\right)^{T} \mathbf{n}_{2}(i)\right\|}{\operatorname{det}\left(\mathbf{A}\left(\mathbf{q}_{\mathbf{k}}\right)^{-1}\right)} C_{G_{\mathbf{R}, k}}\left(f_{T}(\mathbf{x}), i, j\right) .
\end{aligned}
$$

The first term of the intermediate inconsistency function (18) is the derivative of the $3 \mathrm{D}$ radon transform corresponding to the plane, sampled by both projections, calculated on an affine deformation corrected projection of the main scan. The second term is the derivative of the 3D radon transform corresponding to the same plane calculated on a projection of the reference scan. The points $\mathbf{c}_{l, k}$ and $\mathbf{c}_{k, l}$ and the vector $\mathbf{I}_{l}$ are calculated with the geometries $G_{\mathbf{R}, k}^{\prime}$ and $G_{\mathbf{R}, l}$. The sum in (18) runs over a set of unit vectors $\mathbf{l}_{k}(\zeta)$ with $\zeta$ equiangular sampled over $[0, \pi)$. The number of samples of $\zeta$ indicates how many planes are checked for consistency between every pair of projections.

To quantify the inconsistency, the deformation corrected projection is compared with all projections of the reference scan. This results in the following objective function:

$$
F_{k}\left(\mathbf{q}_{k}\right)=\left(\Sigma_{l=1}^{N_{r e f}} T_{k, l}\left(\mathbf{q}_{k}\right)\right)^{\frac{1}{2}}
$$

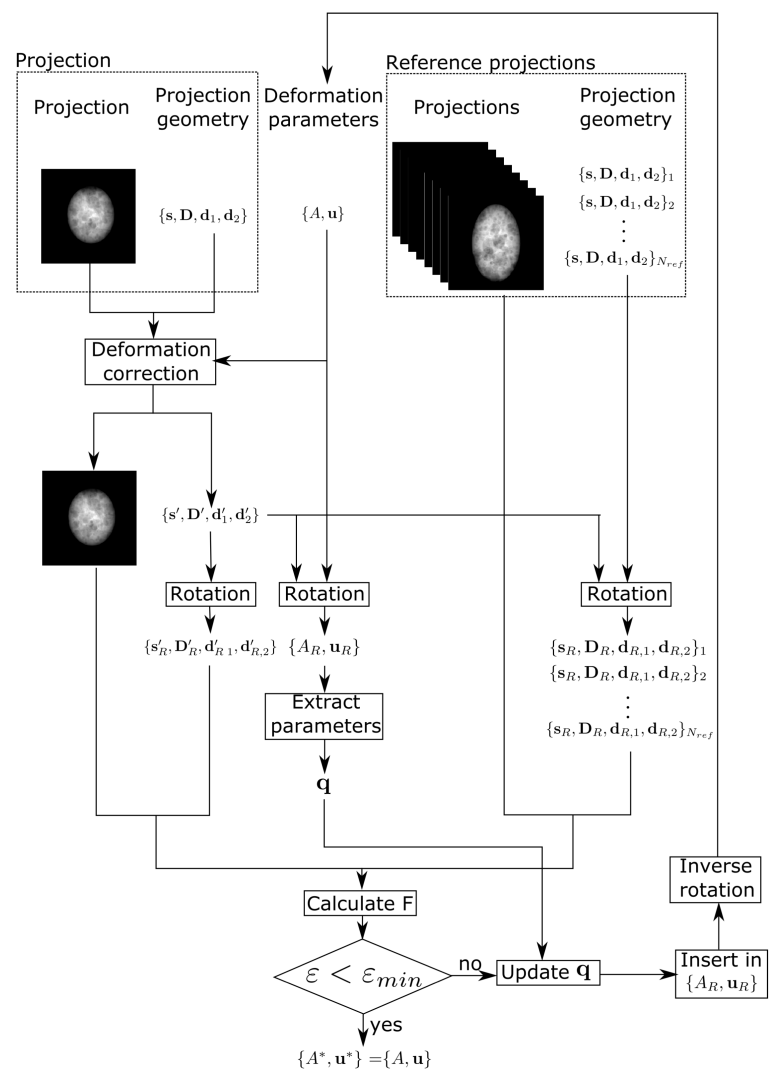

Fig. 4. Schematic overview of the deformation estimation technique for a single projection.

4) Optimization: The objective function in (20) is minimized using a non-linear optimization algorithm:

$$
\mathbf{q}_{k}^{*}=\arg \min _{\mathbf{q}_{k}} F_{k}\left(\mathbf{q}_{k}\right) .
$$

In this work, a Sequential Quadratic Programming (SQP) nonlinear optimization algorithm was used [31]. This procedure is terminated when the number of iterations exceeds $t_{\max }$ or if the relative decrease $\varepsilon$ of $F_{k}$ between two consecutive iterations is below a fixed tolerance, $\varepsilon_{\min }$. These stopping criteria were chosen to be: $\varepsilon_{\min }=10^{-4}$ and $t_{\max }=1000$. A schematic overview is shown in Fig. 4. To avoid local minima, a multi scale approach was applied. To reduce the computational complexity of the algorithm, (4) was approximated as follows

$$
C_{G^{\prime}}(f(\mathbf{x}), i, j) \approx C_{G}\left(f_{T}(\mathbf{x}), i, j\right),
$$

which is approximately valid for small affine deformations.

After the deformation estimation and correction of all projections, the volume is reconstructed at the time of the reference scan. The algorithm was implemented in Matlab, with major parts of the code computed on the Graphics Processing Unit (GPU) using the parallel computing toolbox.

\section{EXPERIMENTS}

The proposed affine deformation estimation and correction technique was validated on both simulated and real data. 


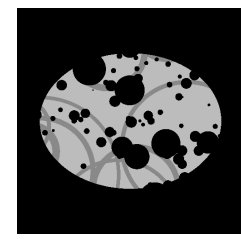

(a)

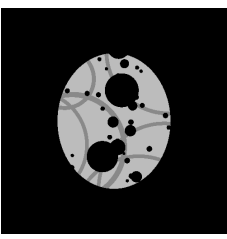

(b)

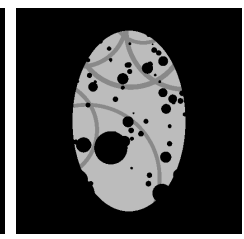

(c)
Fig. 5. Three orthogonal cross-sections of the dough phantom.

\section{A. Phantom Study}

In a first experiment, $8(256 \times 256$ pixels $)$ equiangular projections $\left(360^{\circ}\right.$ scan $)$ for the reference scan were generated from a dough software phantom $(512 \times 512 \times 512$ voxels, see Fig. 5) with the ASTRA-toolbox [32]-[34]. A projection of the affinely deformed phantom was simulated with the following affine deformation parameters:

$$
\begin{aligned}
& \mathbf{A}=\left[\begin{array}{ccc}
1.03 & 0.01 & -0.01 \\
0 & 0.97 & 0.01 \\
0 & 0 & 1.05
\end{array}\right] \\
& \mathbf{u}=[7,-10,-11]^{T} .
\end{aligned}
$$

The performance of the affine deformation estimation method was studied in function of the SNR of the projections, the number of planes that are checked for consistency and the projection angle of the main projection (angle $\phi$ in Fig. 2). Poisson noise was applied to the projection data assuming 20000 photons in the incoming beam per detector pixel (the photon count). Each experiment was repeated 10 times. The estimated and ground truth parameters cannot be compared directly since the proposed technique only estimates deformations perpendicular to the projection direction. The estimated deformation parameters describing these deformations are influenced by deformations in the projection direction. To quantify the quality of the estimated deformation parameters, the mean square error (MSE) was calculated on the projections as follows:

$$
\operatorname{MSE}=\frac{1}{M} \Sigma_{i} \Sigma_{j}\left(C_{\mathbf{q}_{k}^{*}}(f(\mathbf{x}), i, j)-C_{\mathbf{q}_{I d}}\left(f_{T}(\mathbf{x}), i, j\right)\right)^{2},
$$

with:

$$
\begin{aligned}
& C_{\mathbf{q}_{k}}(f(\mathbf{x}), i, j) \\
& \quad=\frac{\operatorname{det}\left(\mathbf{A}^{-1}\right)}{\left\|\left(\mathbf{A}^{-1}\right)^{T} \mathbf{n}_{1}(j)\right\|\left\|\left(\mathbf{A}^{-1}\right)^{T} \mathbf{n}_{2}(i)\right\|} C_{G^{\prime}}(f(\mathbf{x}), i, j) .
\end{aligned}
$$

In (24), $M$ represents the number of pixels on the detector and $\mathbf{q}_{I d}=\left[\begin{array}{lllll}1 & 0 & 0 & 1 & 0\end{array}\right]^{T}$ the parameters of the identity transformation. The MSE compares the noiseless projection of the affine transformed phantom with a projection of the undeformed phantom created with the virtual geometry. This measure reflects the consistency of the corrected projection with the object in the reference state. Since the projections used to calculate the MSE are noiseless, whereas the estimated deformation parameters are calculated on noisy projections, the MSE is a measure of the quality of the estimated deformation parameters.
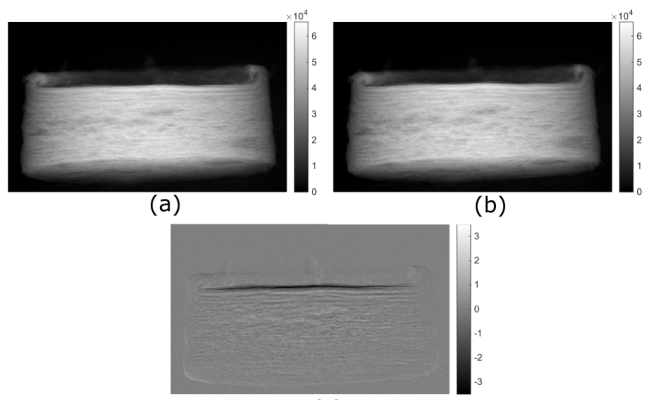

(c)

Fig. 6. The first (a) and last projection (b) of the main scan of the puff pastry dataset, both acquired at the same angle. The difference between these projections is shown in (c).

For the second experiment, the same dough software phantom was used to generate $8(256 \times 256$ pixels $)$ equiangular projections $\left(360^{\circ}\right.$ scan) for the reference scan. The 250 projections of the main scan were generated from an affinely deformed phantom. The affine deformation parameters changed linearly with each projection, starting from the identity deformation and ending with the affine transformation given by (23). For each projection the deformation parameters were estimated. The result of the $(k-1)^{\text {th }}$ projection was used as an initialization of the $k^{\text {th }}$ projection, resulting in a faster estimation. The experiment was repeated for different photon counts.

\section{B. Real Data}

The proposed deformation estimation technique was tested on a CT scan of a leavening puff pastry, acquired with a microCT scanner with a horizontal gantry [35]. Throughout the leavening process the scanner acquired projections during 5 gantry rotations, with a total scan time of 20 minutes. Each gantry rotation consisted of 722 equiangular $401 \times 656$ projections. Each projection was acquired with a source voltage of $60 \mathrm{kV}$, a target current of $200 \mathrm{~mA}$ and an exposure time of $100 \mathrm{~ms}$. Reconstructions, with a voxel size of $12.5 \mu \mathrm{m}$, of each of the rotations were calculated with 300 SIRT iterations. Visual inspection of the reconstruction of the first gantry rotation revealed almost no deformation artefacts. Eight projections of the first gantry rotation, approximately $45^{\circ}$ degrees apart, were selected as reference projections. The next 4 gantry rotations were used to construct the main scan with deformation. One fourth of the projections was selected as to mimic an interleaved scanning protocol (binary decomposition), ensuring maximum angular sampling of the object [36]. Truncation was not observed in the projections (except for the sample holder, which was made of a low attenuating foam). The difference between the first and last projection of the main scan is shown in Fig. 6. The affine deformation parameters were estimated in a two step process. Firstly, only the z-translation and z-scaling were estimated, keeping the rest of the deformation parameters constant at the value of the identity transformation. These two parameters were expected to describe the majority of the deformation. Secondly, all the deformation parameters were estimated with the result of the first estimation as an initial starting point. This strategy guides the estimation in such a way that it avoids local minima in the objective function 


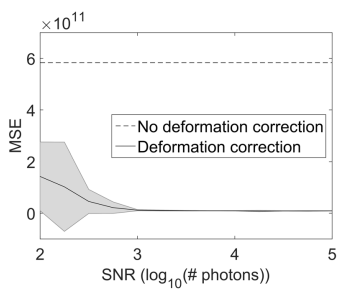

(a)

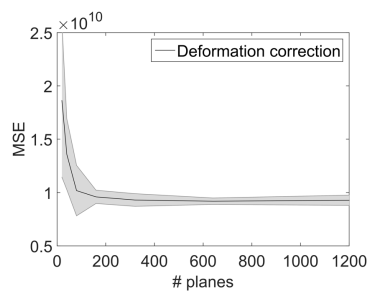

(b)

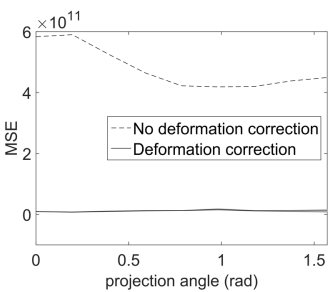

(c)

Fig. 7. MSE values for the dough phantom experiments as a function of (a) the SNR (in terms of photon count), (b) the number of planes compared in every projection pair (the MSE of the projection without deformation correction is $5.83 \times 10^{11}$ ) and (c) the direction of the main projection. The grey areas represent the $95 \%$ confidence interval.

as much as possible. The original and deformation corrected projections (and projection geometries) were reconstructed on a $656 \times 656 \times 401$ voxel grid with 300 SIRT iterations on the distributed version of the ASTRA-toolbox [37], [38].

\section{RESUlts AND Discussion}

\section{A. Phantom Study}

Fig. 7 shows the MSE of the proposed affine deformation correction algorithm in function of the SNR (in terms of the number of photons) (Fig.7a), the number of compared planes between projection pairs (Fig. 7b), and the projection directions (Fig. 7c). The grey areas represents the $95 \%$ confidence interval. Fig. 7a reveals that the algorithm can substantially reduce motion artefacts in terms of the MSE even for very low (as low as 100 photons per detector pixel) photon counts.

In Fig. 7b, the MSE as a function of the number of compared planes between every projection pair is shown. The MSE of the projection without deformation correction is $5.83 \times 10^{11}$, which is significantly higher than all cases with deformation correction. The quality of the estimation improves with the number of compared planes but levels out if more than 200 planes are compared. In general, the optimal number of planes will increase with the number of pixels in the detector.

Fig. 7c shows the MSE for different projection directions with and without deformation estimation. For all projection angles, a clear decrease of the MSE is observed after affine deformation correction. No difference was observed between projections that have an opposing reference projection and projections without opposing reference projection.

In Fig. 8, the reconstructions of the dough phantom simulation experiments are shown for high (left column) and a low (middle column) photon counts. For the low photon count reconstructions, a comparison with the ground truth is provided in Fig. 8 (right column).
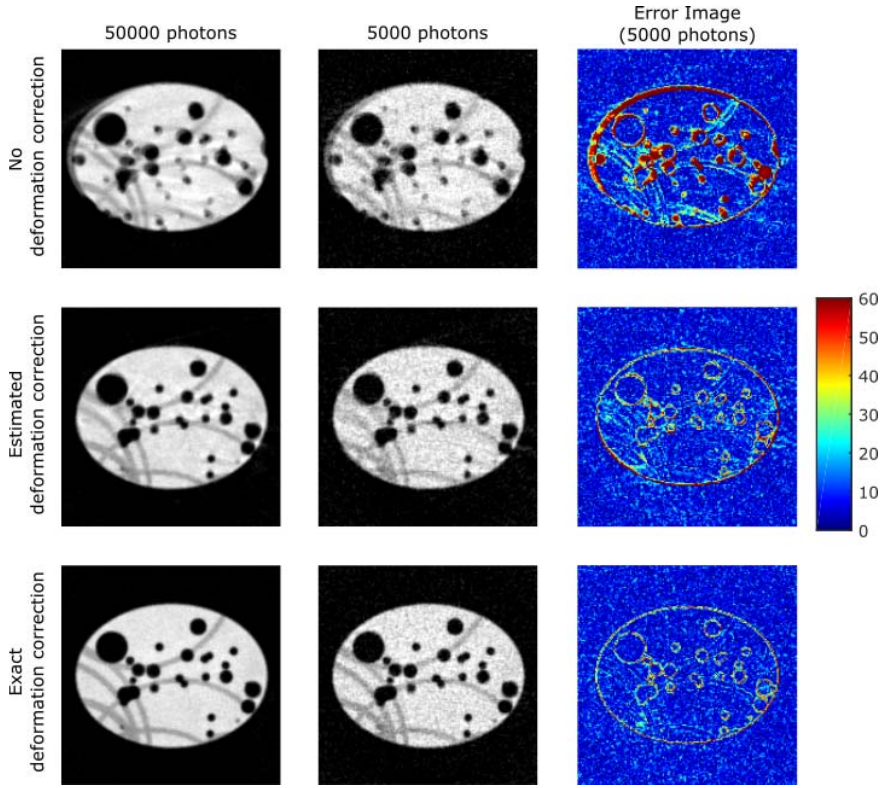

Fig. 8. Reconstructions of a horizontal cross-section (perpendicular to rotation axis) of the dough phantom undergoing affine deformation. The crosssections were motion corrected with different techniques: without deformation correction (top row), with deformation corrected with estimated deformation parameters (estimated deformation correction) (middle row) and with deformation correction with the exact deformation parameters (bottom row). Left column: high photon count (50000 photons per detector pixel). Middle column: low photon count (5000 photons per detector pixel). The images are scaled between 0 and 120. Right column: Absolute difference of the reconstructions (5000 photons per pixel) with the ground truth.

Fig. 8 (top row) shows the reconstructions without deformation correction. The motion artefacts are clearly observable: the borders of the dough and holes are doubled and many structures are substantially blurred. Large deviations from the ground truth are visible in the error image. Fig. 8 (middle row) shows the reconstructions with the proposed affine deformation correction method in which the deformation parameters are estimated from the cone beam projections. As can be observed from these figures, motion artefacts are significantly reduced compared to the reconstructions without deformation correction (top row). In the error image, a close resemblance to the ground truth can be observed. For reference, Fig. 8 (bottom row) also shows the reconstructions after correcting for affine deformation using the (in practice unknown) ground truth deformation parameters. As can be expected, the error image of deformation corrected reconstruction with the ground truth deformation parameters shows the smallest errors.

Because of its short acquisition time, the reference scan provides a set of projections in multiple directions of the (almost undeformed) object. Other strategies could avoid the reference scan and use only the first projection as a reference and optimize the affine deformation parameters in such a way that all projections are consistent. Such a strategy is however flawed in practice. Projections with a projection direction perpendicular to that of the reference projection have only limited redundantly measured data with this reference projection. As a result, the reconstruction can deform in the reference projections projection direction without significantly violating the optimization criterion. The addition of the reference scan solves this problem. 


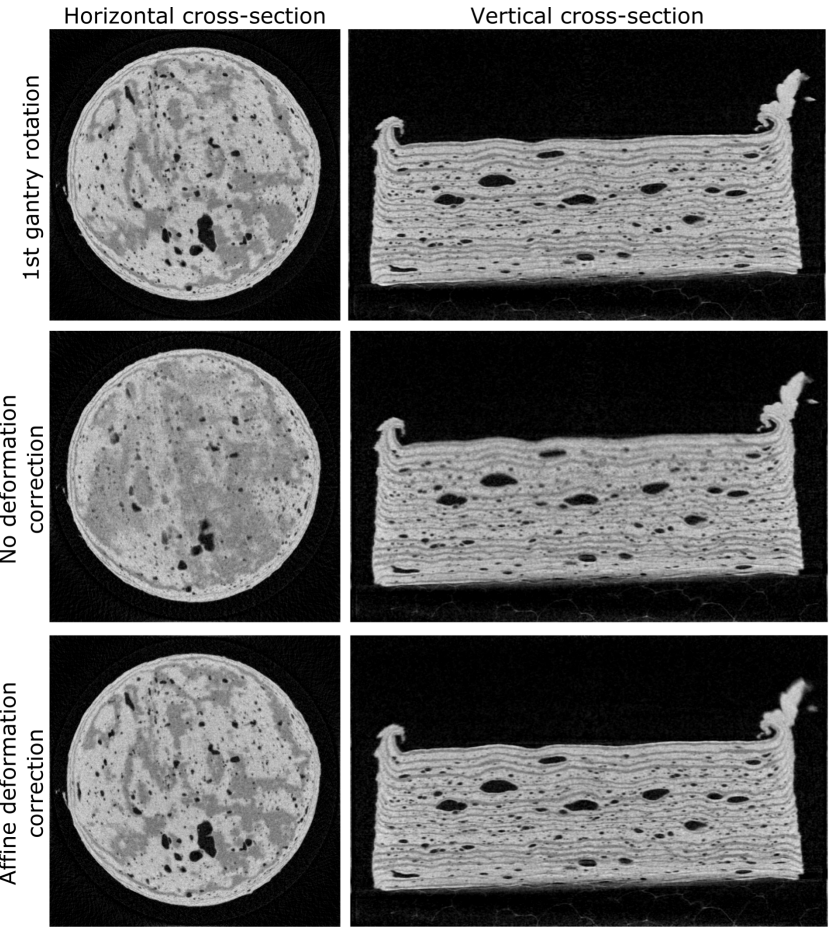

Fig. 9. SIRT reconstructions of the first gantry rotation and reconstructions of the main scan without deformation correction and with deformation correction. Left column: horizontal cross-section (top of object). Right column: vertical cross-section (middle of object).

\section{B. Real Data}

Fig. 9 shows the results of the puff pastry experiment: horizontal and vertical cross-sections are shown in the left and right column, respectively. The reconstructions of the first gantry rotation (Fig. 9 top row) are almost deformation free: a clear separation between the dough layers can be observed and the holes have sharp edges. Fig. 9 (middle row) shows the reconstruction of the main scan without affine deformation correction. This reconstruction is corrupted by motion artefacts. The top of the object is not well reconstructed since this part undergoes the biggest deformation. As a result, gradual transitions between the dough layers can be observed and the borders of the holes are not as sharp as in the first gantry rotation. Fig. 9 (bottom row) shows the reconstructed images of the same dataset with the proposed affine deformation estimation and correction technique. The reconstruction has a high spatial resolution, similar to the reconstruction of the first gantry rotation, though with a much higher SNR. There are clear delineations between the dough layers and the holes have sharp borders. The only part of the reconstruction where motion artefacts are noticeable is at the top right of the vertical cross-section (Fig. 9: third row, right column), where slight blurring of the protruding part can be observed. Fig. 10 shows zoomed images of the reconstructions shown in Fig. 9. Nevertheless, the results of this experiment clearly show that the proposed affine deformation estimation and correction technique performs well in generating almost motion artefact free images. We were able to show that the motion artefacts can be substantially reduced and a better reconstruction quality, with a higher signal to noise ratio, than that achieved by the first gantry rotation.

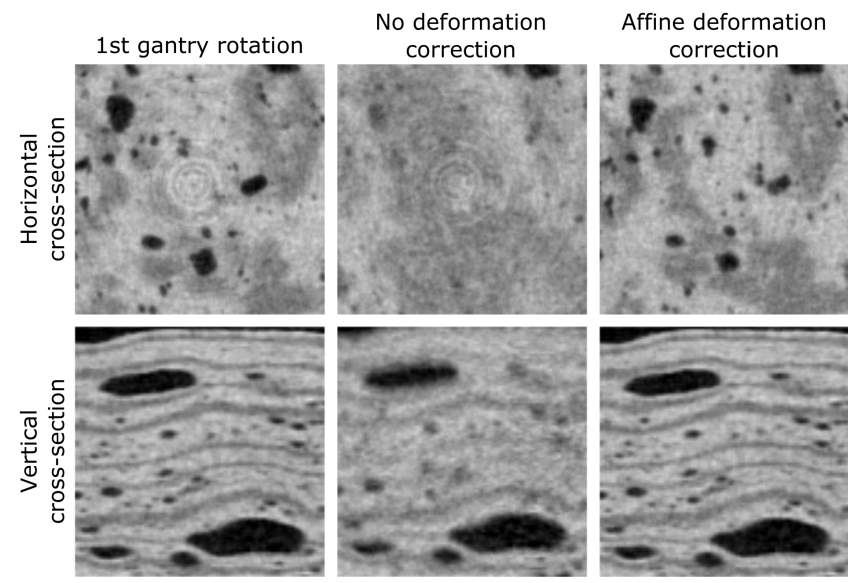

Fig. 10. Zoomed in reconstructions of the first gantry rotation (left column) and reconstructions of the main scan without deformation correction (middle column) and with deformation correction (right column). Top row: horizontal cross-section (Horizontally: top of object. Vertically: middle of object). Bottom row: vertical cross-section (Horizontally: top of object. Vertically: middle of object).

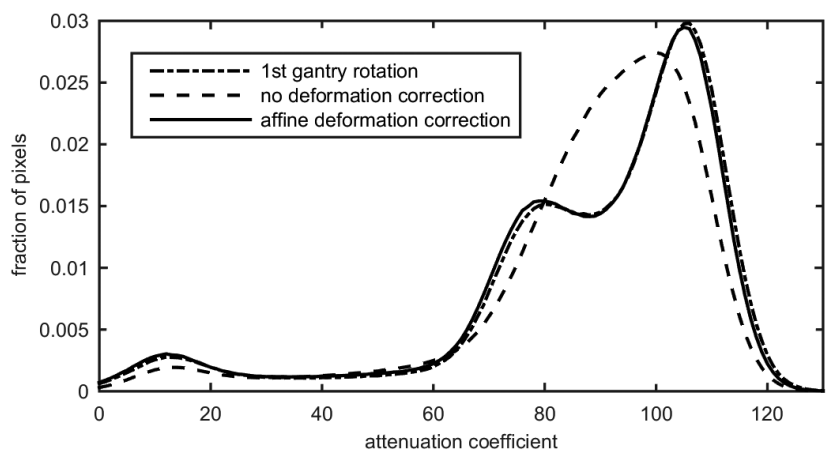

Fig. 11. Histogram of a region of interest (almost the whole puff pastry) of the puff pastry reconstructions.

Motion artefacts have a large effect on the histogram of the reconstruction. Fig. 11 shows the histogram of a region of interest (almost the whole puff pastry without background) in the reconstructions. In the histogram of the first gantry rotation and the deformation corrected reconstruction of the main scan, three modes can be distinguished: a mode corresponding to the holes and two modes corresponding to the different layers in the dough (fat and dough). The histogram of the reconstruction of the main scan without deformation correction shows only two modes. While the holes are still distinguishable in the puff pastry image, the two different dough layers are merged into one broad peak in the histogram. Since the histogram is often used in image post processing, such as determining the thresholds for a segmentation, it is clear that the presence of motion artefacts may significantly influence the results of further analysis.

An interesting (positive) side effect of the proposed motion correction technique is ring artefact reduction, which can be noticed in the reconstructions (see left column Fig. 10). While the horizontal cross-sections of the first gantry rotation and the reconstruction without deformation correction are degraded by ring artefacts, the affine deformation corrected reconstruction shows no ring artefacts at all. This effect is not surprising since 
the origin of these ring artefacts is a combination of a deviating pixel response and the circular trajectory of the gantry [39]. By estimating a virtual projection geometry the source trajectory will no longer be circular and the reconstruction algorithm will no longer produce ring artefacts.

With respect to computational load, the proposed method is efficient in the sense that it does not involve a reconstruction step. The average computation time to estimate the affine deformation of a single projection was only $6.3 \mathrm{~s}$ on a computer with an Intel Core i7-3930K CPU and a NVIDIA GeForce GTX 660 GPU.

\section{4D Reconstructions}

The proposed technique is able to correct for affine deformations during the acquisition and results in a reconstruction at the time point of the reference scan. Often, researchers are interested in 4D reconstructions (3 spatial dimensions and 1 time dimension) of the object and want to visualize the deformation in time. Since an affine deformation is estimated at each projection, a reconstruction at the time point of each projection can be calculated with (3). Nevertheless, the estimated affine deformations are only accurate in the direction orthogonal to the projection direction and, as a result, the visualized deformation may not be in accordance to the real deformation.

This issue can be solved with multiple source-detector pairs. For example, in a scanner with two source-detector pairs, positioned perpendicular with respect to each other, all affine deformation parameters can be estimated, enabling an accurate 4D reconstruction. Alternatively, the deformation parameters corresponding to the projection direction might be estimated by interpolation between the deformation parameters of projections that are as non-parallel as possible and in temporal proximity.

\section{Medical Applications}

The proposed method has numerous applications in micro-CT. In addition, Cone Beam CT (CBCT) is also an important imaging tool in (bio)medical practice. Since the geometry of these acquisitions is equivalent to the geometry in micro-CT, the method can as well be applied in medical CBCT. The affine correction framework can be used for rigid as well as affine motion correction. In applications with only rigid motion (e.g. head motion), the affine parameters corresponding to scaling and shearing are kept constant, while only estimating translation and rotation. Furthermore, our method can even be used as a first order approximation of non-affine motion correction (e.g., respiration), if a reference scan without motion can be acquired.

\section{CONCLUSION}

In this work, an affine deformation estimation and correction technique for cone beam computed tomography was proposed. The proposed method works completely in the projection domain, hence avoiding computationally intensive reconstructions. To correct affine deformations, a relationship between cone beam projections and affine transformations was proven. Estimation of the affine deformation parameters for each projection is achieved by minimizing an inconsistency condition with respect to a fast reference scan consisting of only a few projections.

Experiments on simulated and real data showed that the proposed affine deformation correction method is able to remove or alleviate motion artefacts in non-truncated cone beam projections. Moreover, it reduces ring artefacts as a positive side-effect.

\section{APPENDIX}

In the following section, we will proof (4) and (5). Proof:

$$
\begin{aligned}
C_{G}\left(f_{T}(\mathbf{x}), i, j\right)= & \int f_{T}(\mathbf{x}) \delta\left(\mathbf{n}_{1}(j) \cdot(\mathbf{x}-\mathbf{s})\right) \\
& \times \delta\left(\mathbf{n}_{2}(i) \cdot(\mathbf{x}-\mathbf{s})\right) d \mathbf{x} \\
= & \int f(\mathbf{A x}+\mathbf{u}) \delta\left(\mathbf{n}_{1}(j) \cdot(\mathbf{x}-\mathbf{s})\right) \\
& \times \delta\left(\mathbf{n}_{2}(i) \cdot(\mathbf{x}-\mathbf{s})\right) d \mathbf{x}
\end{aligned}
$$

Change of variables $\mathbf{y}=\mathbf{A x}+\mathbf{u}$ :

$$
\begin{aligned}
C_{G}\left(f_{T}(\mathbf{x}), i, j\right) \\
=\operatorname{det}\left(\mathbf{A}^{-1}\right) \int f(\mathbf{y}) \delta\left(\mathbf{n}_{1}(j) \cdot\left(\mathbf{A}^{-1}(\mathbf{y}-\mathbf{u})-\mathbf{s}\right)\right) \\
\quad \times \delta\left(\mathbf{n}_{2}(i) \cdot\left(\mathbf{A}^{-1}(\mathbf{y}-\mathbf{u})-\mathbf{s}\right)\right) d \mathbf{y}
\end{aligned}
$$

Since $\mathbf{A x} \cdot \mathbf{y}=\mathbf{x} \cdot \mathbf{A}^{T} \mathbf{y}$ and $\delta(x)=|a| \delta(a x)$, we have:

$$
\begin{aligned}
C_{G}( & \left.f_{T}(\mathbf{x}), i, j\right) \\
= & \frac{\operatorname{det}\left(\mathbf{A}^{-1}\right)}{\left\|\left(\mathbf{A}^{-1}\right)^{T} \mathbf{n}_{1}(j)\right\|\left\|\left(\mathbf{A}^{-1}\right)^{T} \mathbf{n}_{2}(i)\right\|} \\
& \times \int f(\mathbf{y}) \delta\left(\frac{\left(\mathbf{A}^{-1}\right)^{T} \mathbf{n}_{1}(j)}{\left\|\left(\mathbf{A}^{-1}\right)^{T} \mathbf{n}_{1}(j)\right\|} \cdot(\mathbf{y}-\mathbf{u}-\mathbf{A} \mathbf{s})\right) \\
& \times \delta\left(\frac{\left(\mathbf{A}^{-1}\right)^{T} \mathbf{n}_{2}(i)}{\left\|\left(\mathbf{A}^{-1}\right)^{T} \mathbf{n}_{2}(i)\right\|} \cdot(\mathbf{y}-\mathbf{u}-\mathbf{A s})\right) d \mathbf{y} .
\end{aligned}
$$

Next, a virtual geometry is defined as:

$$
\begin{aligned}
& \left\{\begin{array}{l}
\mathbf{s}^{\prime}=\mathbf{A s}+\mathbf{u} \\
\mathbf{d}_{c}^{\prime}=\mathbf{A} \mathbf{d}_{c}+\mathbf{u} \\
\mathbf{d}_{1}^{\prime}=\mathbf{A} \mathbf{d}_{1} \\
\mathbf{d}_{2}^{\prime}=\mathbf{A} \mathbf{d}_{2}
\end{array}\right. \\
C_{G}\left(f_{T}(\mathbf{x}), i, j\right)= & \frac{\operatorname{det}\left(\mathbf{A}^{-1}\right)}{\left\|\left(\mathbf{A}^{-1}\right)^{T} \mathbf{n}_{1}\right\|\left\|\left(\mathbf{A}^{-1}\right)^{T} \mathbf{n}_{2}\right\|} \int f(\mathbf{y}) \\
& \times \delta\left(\frac{\left(\mathbf{A}^{-1}\right)^{T} \mathbf{n}_{1}(j)}{\left\|\left(\mathbf{A}^{-1}\right)^{T} \mathbf{n}_{1}(j)\right\|} \cdot\left(\mathbf{y}-\mathbf{s}^{\prime}\right)\right) \\
& \times \delta\left(\frac{\left(\mathbf{A}^{-1}\right)^{T} \mathbf{n}_{2}(i)}{\left\|\left(\mathbf{A}^{-1}\right)^{T} \mathbf{n}_{2}(i)\right\|} \cdot\left(\mathbf{y}-\mathbf{s}^{\prime}\right)\right) d \mathbf{y} .
\end{aligned}
$$


We will now show that $\frac{\left(\mathbf{A}^{-1}\right)^{T} \mathbf{n}_{1}(j)}{\left\|\left(\mathbf{A}^{-1}\right)^{T} \mathbf{n}_{1}(j)\right\|}=\mathbf{n}^{\prime}{ }_{1}(j)$ :

$$
\begin{aligned}
\frac{\left(\mathbf{A}^{-1}\right)^{T} \mathbf{n}_{1}(j)}{\left\|\left(\mathbf{A}^{-1}\right)^{T} \mathbf{n}_{1}(j)\right\|} & =\frac{1}{Z}\left(\mathbf{A}^{-1}\right)^{T}\left[\left(\mathbf{m}_{i j}-\mathbf{s}\right) \times \mathbf{d}_{1}\right] \\
& =\frac{\operatorname{det}(\mathbf{A})}{Z}\left[\mathbf{A}\left(\mathbf{d}_{c}+j \mathbf{d}_{2}-\mathbf{s}\right) \times \mathbf{A} \mathbf{d}_{1}\right] \\
& =\frac{1}{Z^{\prime}}\left[\left(\mathbf{d}_{c}^{\prime}+j \mathbf{d}_{2}^{\prime}-\mathbf{s}^{\prime}\right) \times \mathbf{d}_{1}^{\prime}\right] \\
& =\mathbf{n}_{1}^{\prime}(j) .
\end{aligned}
$$

Here we used the property: $\mathbf{A}(\mathbf{x} \times \mathbf{y})=\operatorname{det}\left(\mathbf{A}^{-1}\right)$ $\left[\left(\mathbf{A}^{-1}\right)^{T} \mathbf{x} \times\left(\mathbf{A}^{-1}\right)^{T} \mathbf{y}\right] . Z$ and $Z^{\prime}$ are normalization constants. We can prove equivalently that $\frac{\left(\mathbf{A}^{-1}\right)^{T} \mathbf{n}_{2}(i)}{\left\|\left(\mathbf{A}^{-1}\right)^{T} \mathbf{n}_{2}(i)\right\|}=\mathbf{n}_{2}^{\prime}(i)$. Substituting these relations in (30), we have:

$$
\begin{aligned}
& C_{G}\left(f_{T}(\mathbf{x}), i, j\right) \\
& =\frac{\operatorname{det}\left(\mathbf{A}^{-1}\right)}{\left\|\left(\mathbf{A}^{-1}\right)^{T} \mathbf{n}_{1}(j)\right\|\left\|\left(\mathbf{A}^{-1}\right)^{T} \mathbf{n}_{2}(i)\right\|} \int f(\mathbf{y}) \delta\left(\mathbf{n}_{1}^{\prime}(j) \cdot\left(\mathbf{y}-\mathbf{s}^{\prime}\right)\right) \\
& \quad \times \delta\left(\mathbf{n}_{2}^{\prime}(i) \cdot\left(\mathbf{y}-\mathbf{s}^{\prime}\right)\right) d \mathbf{y} .
\end{aligned}
$$

If we compare (32) with (2), we can write:

$$
\begin{aligned}
& C_{G}\left(f_{T}(\mathbf{x}), i, j\right) \\
& \quad=\frac{\operatorname{det}\left(\mathbf{A}^{-1}\right)}{\left\|\left(\mathbf{A}^{-1}\right)^{T} \mathbf{n}_{1}(j)\right\|\left\|\left(\mathbf{A}^{-1}\right)^{T} \mathbf{n}_{2}(i)\right\|} C_{G^{\prime}}(f(\mathbf{x}), i, j),
\end{aligned}
$$

with $G^{\prime}=\left\{\mathbf{s}^{\prime}, \mathbf{d}_{c}^{\prime}, \mathbf{d}_{1}^{\prime}, \mathbf{d}_{2}^{\prime}\right\}$.

\section{ACKNOWLEDGMENT}

The authors would like to thank Lantmännen Unibake Belgium for providing the puff pastry. Thanks to Prof. A. J. den Dekker for careful proofreading.

\section{REFERENCES}

[1] C. J. Ritchie, J. D. Godwin, C. R. Crawford, W. Stanford, H. Anno, and Y. Kim, "Minimum scan speeds for suppression of motion artifacts in CT," Radiology, vol. 185, no. 1, pp. 37-42, 1992.

[2] G. Van Eyndhoven et al., "An iterative CT reconstruction algorithm for fast fluid flow imaging," IEEE Trans. Image Process., vol. 24, no. 11, pp. 4446-4458, Nov. 2015.

[3] W. Lu, P. J. Parikh, J. P. Hubenschmidt, J. D. Bradley, and D. A. Low, "A comparison between amplitude sorting and phase-angle sorting using external respiratory measurement for 4D CT," Med. Phys., vol. 33, no. 8, pp. 2964-2974, Jul. 2006.

[4] M. Grass et al., "Helical cardiac cone beam reconstruction using retrospective ECG gating," Phys. Med. Biol., vol. 48, no. 18, pp. 3069-3084, Sep. 2003.

[5] A. A. Isola, A. Ziegler, T. Koehler, W. J. Niessen, and M. Grass, "Motion-compensated iterative cone-beam CT image reconstruction with adapted blobs as basis functions," Phys. Med. Biol., vol. 53, no. 23, pp. 6777-6797, 2008.

[6] S. Rit, D. Sarrut, and L. Desbat, "Comparison of analytic and algebraic methods for motion-compensated cone-beam CT reconstruction of the thorax," IEEE Trans. Med. Imag., vol. 28, no. 10, pp. 1513-1525, Oct. 2009.

[7] S. Roux, L. Desbat, A. Koenig, and P. Grangeat, "Exact reconstruction in 2D dynamic CT: Compensation of time-dependent affine deformations," Phys. Med. Biol., vol. 49, no. 11, pp. 2169-2182, Jun. 2004.

[8] L. Desbat, S. Roux, and P. Grangeat, "Compensation of some time dependent deformations in tomography," IEEE Trans. Med. Imag., vol. 26, no. 2, pp. 261-269, Feb. 2007.

[9] J.-H. Kim, J. Nuyts, A. Kyme, Z. Kuncic, and R. Fulton, "A rigid motion correction method for helical computed tomography (CT)," Phys. Med. Biol., vol. 60, pp. 2047-2073, Feb. 2015.

[10] J.-H. Kim, T. Sun, A. R. Alcheikh, Z. Kuncic, J. Nuyts, and R. Fulton, "Correction for human head motion in helical X-ray CT," Phys. Med. Biol., vol. 61, no. 4, pp. 1416-1438, Feb. 2016.
[11] C. P. V. Christoffersen, D. Hansen, P. Poulsen, and T. S. Sorensen, "Registration-based reconstruction of four-dimensional cone beam computed tomography," IEEE Trans. Med. Imag., vol. 32, no. 11, pp. 2064-2077, Nov. 2013.

[12] A. A. Isola, M. Grass, and W. J. Niessen, "Fully automatic nonrigid registration-based local motion estimation for motion-corrected iterative cardiac CT reconstruction," Med. Phys., vol. 37, no. 3, pp. 1093-1109, Mar. 2010.

[13] Q. Tang, J. Cammin, S. Srivastava, and K. Taguchi, "A fully fourdimensional, iterative motion estimation and compensation method for cardiac CT," Med. Phys., vol. 39, no. 7, pp. 4291-4305, May 2012.

[14] C. Rolf and D. Laurent, "Data consistency conditions for truncated fanbeam and parallel projections," Med. Phys., vol. 42, no. 2, pp. 831-845, Feb. 2015.

[15] M. Negahdar and A. A. Amini, "Estimation of affine motion from projection data using a mass conservation model," in Proc. 33rd Annu. Int. Conf. IEEE EMBS, Jan. 2011, pp. 8041-8044.

[16] R. Mooser, F. Forsberg, E. Hack, G. Székely, and U. Sennhauser, "Estimation of affine transformations directly from tomographic projections in two and three dimensions," Mach. Vis. Appl., vol. 24, no. 2, pp. 419-434, Oct. 2011.

[17] S. R. Deans, The Radon Transform and Some of Its Applications. North Chelmsford, MA, USA: Courier Corporation, 2007.

[18] X. Xiong and K. Qin, "Linearly estimating all parameters of affine motion using radon transform," IEEE Trans. Image Process., vol. 23, no. 10, pp. 4311-4321, Oct. 2014.

[19] R. Frysch and G. Rose, "Rigid motion compensation in interventional C-arm CT using consistency measure on projection data," in Proc. MICCAI, vol. 9349. 2015, pp. 298-306.

[20] W. Wein and A. Ladikos, "Towards general motion recovery in conebeam computed tomography," in Proc. Fully Three-Dimensional Image Reconstruct. Radiol. Nucl. Med., Jun. 2013, pp. 54-57.

[21] S. Leng, B. Nett, M. Speidel, and G.-H. Chen, "Motion artifact reduction in fan-beam and cone-beam computed tomography via the fan-beam data consistency condition (FDCC)," Proc. SPIE, vol. 6510, p. 65101W, Mar. 2007.

[22] P. Grangeat, "Mathematical framework of cone beam 3D reconstruction via the first derivative of the radon transform," in Mathematical Methods in Tomography, vol. 1497. Berlin, Germany: Springer, 1990, pp. 66-97.

[23] M. Defrise and R. Clack, "A cone-beam reconstruction algorithm using shift-variant filtering and cone-beam backprojection," IEEE Trans. Med. Imag., vol. 13, no. 1, pp. 186-195, Mar. 1994.

[24] C. Debbeler, N. Maass, M. Elter, F. Dennerlein, and T. Buzug, "A new CT raw data redundancy measure applied to automated misalignment correction," in Proc. Fully Three-Dimensional Image Reconstruct. Radiol. Nucl. Med., 2013, pp. 264-267.

[25] M. Elter, N. Maass, and P. Koch, "Suitability of a new alignment correction method for industrial CT," in Proc. Conf. Ind. Comput. Tomogr., Feb. 2014, pp. 245-252.

[26] A. Aichert et al., "Redundancies in X-ray images due to the epipolar geometry for transmission imaging," in Proc. 3th Int. Conf. Image Formation X-Ray Comput. Tomogr., 2015, pp. 333-337.

[27] A. Aichert et al., "Epipolar consistency in transmission imaging," IEEE Trans. Med. Imag., vol. 34, no. 11, pp. 2205-2219, Nov. 2015.

[28] P. L. Salmon, X. Liu, and A. Sasov, "A post-scan method for correcting artefacts of slow geometry changes during micro-tomographic scans," $J$. X-Ray Sci. Technol., vol. 17, no. 2, pp. 161-174, Feb. 2009.

[29] T. Grulich, W. Holub, U. Haßler, A. Aichert, and A. Maier, "Geometric adjustment of X-ray tomosynthesis," in Proc. Fully Three-Dimensional Image Reconstruct. Radiol. Nucl. Med., Jun. 2015, pp. 468-471.

[30] L. A. Feldkamp, L. C. Davis, and J. W. Kress, "Practical cone-beam algorithm," J. Opt. Soc. Amer. A, vol. 1, no. 6, pp. 612-619, Jun. 1984.

[31] J. Nocedal and S. J. Wright, Numerical Optimization (Springer Series in Operations Research). New York, NY, USA: Springer-Verlag, 2006.

[32] W. J. Palenstijn, K. J. Batenburg, and J. Sijbers, "Performance improvements for iterative electron tomography reconstruction using graphics processing units (GPUs)," J. Struct. Biol., vol. 176, no. 2, pp. 250-253, Nov. 2011.

[33] W. van Aarle et al., "The ASTRA toolbox: A platform for advanced algorithm development in electron tomography," Ultramicroscopy, vol. 157, pp. 35-47, Oct. 2015.

[34] W. van Aarle et al., "Fast and flexible X-ray tomography using the ASTRA toolbox," Opt. Exp., vol. 24, no. 22, pp. 25129-25147, 2016.

[35] M. Dierick et al., "Recent micro-CT scanner developments at UGCT," Nucl. Instrum. Methods Phys. Res. B, Beam Interact. Mater. At., vol. 324, pp. 35-40, Apr. 2014. 
[36] A. P. Kaestner, B. Münch, P. Trtik, and L. Butler, "Spatiotemporal computed tomography of dynamic processes," Opt. Eng., vol. 50, no. 12, p. 123201, Dec. 2011.

[37] J. Gregor and T. Benson, "Computational analysis and improvement of SIRT,” IEEE Trans. Med. Imag., vol. 27, no. 7, pp. 918-924, Jul. 2008.

[38] W. J. Palenstijn, J. Bédorf, and K. J. Batenburg, "A distributed SIRT implementation for the ASTRA toolbox," in Proc. Fully ThreeDimensional Image Reconstruct. Radiol. Nucl. Med., Jun. 2015, pp. 166-169.

[39] J. Sijbers and A. Postnov, "Reduction of ring artefacts in high resolution micro-CT reconstructions," Phys. Med. Biol., vol. 49, no. 14, pp. 247-253, Jul. 2004.

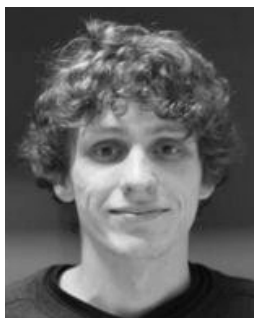

Vincent Van Nieuwenhove received the M.Sc. degree in physics from the University of Antwerp, Belgium, in 2013, where he is currently pursuing the Ph.D. degree with the imec-Vision Laboratory, Department of Physics. His Ph.D. research mainly focuses on reconstruction methods for dynamic computed tomography and advanced flat field correction in X-ray imaging.

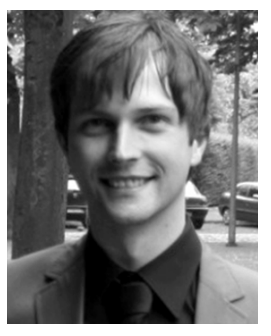

Jan De Beenhouwer received the M.Sc. degree in computer science engineering from KU Leuven, Belgium, in 2003, and the Ph.D. degree in biomedical engineering from Ghent University, Belgium, in 2008. He was a Post-Doctoral Fellow for two years at the same institution prior to joining the imec-Vision Lab, University of Antwerp, Belgium, where he is currently active as research leader of the ASTRA tomography group. His main interest is in image reconstruction with focus on X-ray computed tomography and electron tomography.

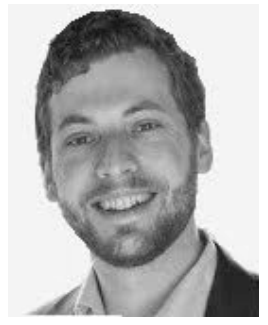

Thomas De Schryver received the master's degrees in mechanical engineering and applied physics from Ghent University, Belgium, in 2008 and 2012, respectively. From 2008 to 2010, he was a Researcher with the BioMMeda Research Group, Ghent University, where he was involved in the numerical models of the human vasculature system. From 2013 to 2017, he was a Doctoral Researcher with the Centre for X-Ray Tomography, Ghent University. Pending his Ph.D. defense, he is currently with the X-Ray Engineering, Belgium, performing research and development towards custom CT scanner design and CT advanced reconstruction algorithms.

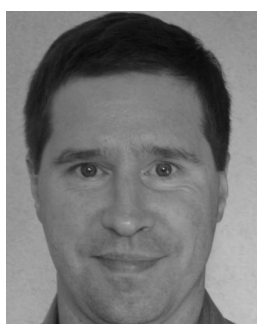

Luc Van Hoorebeke received the Licentiate degree in physics and the Ph.D. degree in physics from Ghent University, Belgium, in 1985 and 1991, respectively. During 1993 and 1994, he was a Researcher with the Belgian Nuclear Research Centre, where he was involved in the field of positron annihilation spectroscopy. He was with Ghent University as a Post-Doctoral Fellow of the Research Foundation - Flanders in the field of experimental nucleon physics. In 2003, he was a Professor of Physics with the Department of Physics and Astronomy, Ghent University, and also the Head of the Radiation Physics Research Group. In 2006, he co-founded the Centre for X-Ray Tomography, Ghent University. His current research topic is high resolution X-ray tomography.

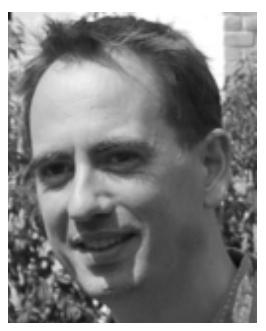

Jan Sijbers received the M.Sc. degree in physics and the Ph.D. degree in sciences/physics from the University of Antwerp, in 1993 and 1998, respectively. He was a Post-Doctoral Fellow of the Fund for Scientific Research from 2002-2008, jointly with the University of Antwerp and the Delft University of Technology, The Netherlands. In 2010, he was appointed as a Senior Lecturer and in 2014, he became Full Professor with the University of Antwerp. He has (co)authored over 220 journal papers. His main interest is in biomedical image reconstruction, processing and analysis with focus on magnetic resonance processing and iterative reconstruction for computed tomography. 